\title{
Asociación del sobrepeso y obesidad, consumo de oxígeno y calidad del sueño con los síntomas del intestino irritable en estudiantes universitarios de la UPNFM
}

\author{
Association of physical state, oxygen consumption and sleep \\ quality with the irritable bowel symptoms in university students \\ of the UPNFM
}

* Aldo Alfonso Vasquez Bonilla

Vasquez, A. (2019). Asociación del sobrepeso y obesidad, consumo de oxígeno y calidad del sueño con los síntomas del intestino irritable en estudiantes universitarios de la UPNFM. Revista Ciencias de la Actividad Física UCM, N²0(2), julio-diciembre, 1-19. DOl: http://doi.org/10.29035/rcaf.20.2.6

\section{RESUMEN}

Introducción: El interés de este estudio fue establecer posibles asociaciones con el estado físico, calidad de sueño y consumo de oxígeno máximo $\left(\mathrm{VO}_{2}\right.$ máx) con los síntomas de la enfermedad del síndrome de Intestino Irritables (SII) en los estudiantes universitarios.

Métodos: La muestra fue de $n=62$ estudiantes universitarios (edad $25 \pm 9$ años; talla 1,63 $\pm 0,08 \mathrm{~m}$; peso $68,6 \pm 18,5 \mathrm{~kg}$ ). Se midieron variables antropométricas, el $\mathrm{VO}_{2}$ máx mediante el test Rockport, se aplicó el cuestionario de índice de Calidad de Sueño de "Pittsburgh" (ICSP) y el "BEST-Score" 4 points. Se utilizó la prueba Anova y el coeficiente de Pearson.

Resultado: En los síntomas del SII (\%) se muestran diferencias entre sujetos normopeso vs obesidad (17,5 \pm 10 vs 33,3 $\pm 23,1 \% \mathrm{p}=0.00)$. También se muestra diferencias en función del $\mathrm{VO}_{2}$ máx en ICPS: nivel 7 vs nivel 3 (26 \pm 0,6 vs $13 \pm 0,6 \%$ $\mathrm{p}=0.04)$ y síntomas del SII (21,9 $\pm 10 \mathrm{vs} 11,1 \pm 17,9 \% \mathrm{p}=0.02)$. Los síntomas del SII obtuvieron correlación moderada con el IMC ( $r=0,57 ; p=0,00)$ y \% de grasa ( $r=$ $0,60 ; p=0,00)$, también el $\mathrm{VO}_{2}$ máx con la calidad del sueño $(r=-0,52 ; p=0,00$ ). 
Conclusión: Los estudiantes de la UPNFM que presentan un mayor $\mathrm{VO}_{2}$ máx muestran valores más bajos en la puntuación de síntomas del SII y podría estar asociado a conciliar mejor el sueño.

\section{Palabras clave}

Medicina preventiva, salud pública, estudiante universitario, obesidad y fisiología humana.

\section{ABSTRACT}

Introduction: The interest of this study was to establish possible associations with the physical state, sleep quality and maximum oxygen consumption $\left(\mathrm{VO}_{2} \mathrm{max}\right)$ with the symptoms of Irritable Bowel Syndrome (IBS) in university students.

Methods: The sample was $n=62$ university students (age $25 \pm 9$ years, height $1.63 \pm 0.08 \mathrm{~m}$, weight $68.6 \pm 18.5 \mathrm{~kg}$ ). Anthropometric variables were measured, $\mathrm{VO}_{2}$ max using the Rockport test, the "Pittsburgh Quality" Sleep Quality Index questionnaire (ICSP) and the "BEST-Score" 4 points were applied. The Anova test and the Pearson coefficient were used.

Result: In the IBS symptoms (\%) there are differences between subject's normal weight vs obesity $(11.5 \pm 10$ vs $33.3 \pm 23.1 \% p=0.00)$. It also shows differences according to $\mathrm{VO}_{2}$ max in ICPS: level 1 vs level 3 ( $26 \pm 0.6$ vs $13 \pm 0.6 \% \mathrm{p}=0.04$ ) and IBS symptoms (21.9 \pm 10 vs $11.1 \pm 17,9 \% \mathrm{p}=0.02)$. The IBS symptoms showed moderate correlation with BMI ( $r=0.51, p=0.00)$ and of fat\% $(r=0.60, p=0.00)$, also the $\mathrm{VO}_{2}$ max with the quality of sleep ( $\left.r=-0.52, p=0.00\right)$.

Conclusion: Students of UPNFM who present a higher $\mathrm{VO}_{2}$ max show lower values in the symptom score of IBS and could be associated to better sleep conciliation.

\section{Key words}

Medicine prevention, Public health, University students, Obesity and Human physiology. 


\section{Introducción}

La enfermedad del síndrome de intestino irritable (SII) es uno de los trastornos digestivos funcionales más frecuentes, dado que su prevalencia se estima entre el 5\% y el 15\% en la población mundial (Quigley, Abdel-Hamid, Barbara, Bhatia, Boeckxstaens, De Giorgio, et al., 2012). EI SII está asociado a costos de salud altos (Talley, Gabriel, Harmsen, Zinsmeister \& Evans, 1995) pues su diagnóstico no es fácil, aunque las causas de este síndrome no se conocen bien, hace tiempo se sabe que está relacionado con el estado psicológico del paciente como la ansiedad y depresión (Cerecedo, 2011). El coste asociado al manejo de la enfermedad se deriva principalmente del elevado número de visitas médicas, lo que evidencia necesidades no cubiertas en el manejo terapéutico de dichos pacientes (Cortés, Roset, Barrull, Sais \& Raya, 2016).

El diagnóstico del colon irritable se realiza por exclusión, ya que no se dispone de exámenes específicos para confirmar la presencia de este síndrome, siendo los criterios del Roma III los más utilizados en la cuantificación de los síntomas individuales, ya que parece reflejar mejor el diagnóstico clínico de SII aplicado por médicos (Drossman, 2006). También se utilizan ensayos/cuestionarios de investigación que componen varias dimensiones del dolor, como la intensidad, constancia, frecuencia y predictibilidad (Spiegel, Bolus, Harris, Lucak, Chey, Sayuk, et al., 2010).

EI SII afecta la calidad de vida en algunos estudiantes universitarios por los niveles de ansiedad producidos (Alaqeel, Alowaimer, Alonezan, Almegbel \& Alaujan, 2017), esto podría verse reflejado en un aumento del SII, ya que es un trastorno que puede prevalecer en una población universitaria de entre 18 y 30 años y tiene un impacto significativo en la calidad de vida (Cañón, Ruiz, Rondón \& Alvarado, 2017)

En el contexto del SII hay autores que proponen la variable Actividad Física en diferentes estudios como parte del tratamiento integral, por ejemplo, en el estudio de Foster, Hillsdon, Thorogood \& Kaur (2014) se identificó que la mayor frecuencia de actividad física y el incremento de oxígeno tiene efectos positivos en la disminución de sintomatología del SII, sobre todo en individuos con obesidad, diabetes mellitus tipo 2 e hipertensión que suelen estar asociados al SII. Por lo tanto, la actividad física de intensidad moderada conlleva muchos beneficios en sujetos con problemas gastrointestinales (Johannesson, Ringstrom, Abrahamsson \& Sadik, 2015), sobre todo en los que presentan estreñimiento predominante asociado al SII (De Schryver, Keulemans, Peters, Akkermans, Smout, De Vries, et al., 2005; Song, Cho, Jo, Oh \& Kim, 2012). 
Por ello, se indagaron las posibles asociaciones entre el $\mathrm{VO}_{2}$ máx, calidad del sueño y obesidad para la prevención de futuros problemas relacionados con sintomatología del SII en estudiantes universitarios hondureños; estos datos pueden ser utilizados en la prevención de esta patología en población universitaria, y no deben estar olvidados en la promoción de la salud (Ledo-Varela, de Luis, GonzálezSagrado, Izaola-Jauregui, Conde-Vicente \& Aller, 2011).

\section{Metodología}

\section{Tipo de Investigación}

Este estudio se desarrolló con una metodología cuantitativa no-experimental de tipo descriptivo-comparativo y de diseño transversal.

\section{Participantes}

La muestra estuvo constituida por 62 estudiantes universitarios de diferentes carreras que asistían a las clases de Educación Física; del total, n = 37 fueron mujeres (56\%) y $n=29$ fueron hombres (44\%). Las características de la muestra se observan en la Tabla 1, expresando los datos como la media \pm desviación estándar. Se excluyeron del estudio los sujetos que tenían una medicación que pudiese afectar la calidad de sueño, la prueba de $\mathrm{VO}_{2}$ máx y el SII. Las mujeres que informaron embarazo fueron excluidas del estudio. Se incluyeron los sujetos que estaban matriculados en cada una de sus respectivas facultades. Todos los participantes firmaron un consentimiento informado de participación voluntaria, de conformidad con los principios de la Declaración de Helsinki.

\section{Mediciones e Instrumentos}

Mediciones antropométricas: para caracterizar el estado físico de los individuos se realizaron mediciones del Índice de Masa Corporal (IMC), Índice Cintura-Cadera (ICC), Masa Grasa \% e Índice Adiposidad Corporal (IAC). La altura del cuerpo se midió usando un estadiómetro portátil (Seca 213, Alemania) y el peso usando una balanza Tanita Body Composition Monitor UM-08, el IMC se calculó a partir de la relación peso / altura2 ( $\left.\mathrm{kg} / \mathrm{m}^{2}\right)$. Para medir el ICC se utilizó una cinta métrica que registra circunferencias con una precisión de $0,1 \mathrm{~cm}$, se obtuvo la media de dos mediciones aplicando la formula $\mathrm{ICC}=$ Cintura/Cadera. Se tomó como punto de corte un resultado $\geq 0,50$ (Browning, Hsieh \& Ashwell, 2010). La Masa Grasa se evaluó mediante bioimpedancia eléctrica (AIB), se ajustó mediante la altura, peso, edad y sexo. Se midió con el Body Composition monitor (Hbf-306int de OMRON, USA), la medición se realizó con las normas correspondientes propuestas en el estudio de (Alvero-Cruz, Correas, Ronconi, Fernández \& Porta i Manzañido, 2011). El IAC se estimó con la ecuación propuesta por Bergman, Stefanovski, Buchanan, Sumner, Rey- 
nolds, Sebring, et al., 2011: IAC = [(Perímetro de Cadera, en cm)/((altura, en m)1,5 )-18)].

\section{Consumo de Oxígeno Máximo}

$\left(\mathrm{VO}_{2}\right.$ máx): para estimar el $\mathrm{VO}_{2}$ máx se utilizó el test de Rockport, donde los sujetos recorrieron una distancia de 1609 m. en la pista polideportiva. Una vez se completó la distancia marcada, se anotó el tiempo empleado por cada uno de los sujetos y 15 segundos después se mencionó el número de pulsaciones con las que se terminó la prueba.

La frecuencia cardíaca máxima se evaluó con el monitor de frecuencia de pulso Polar Polar@ S810i y se procesó con el software Polar Precision Performance 3.0 (versión 4.00.020).

Estos datos y otros personales (edad, sexo y peso) deberán introducirse en una fórmula para obtener la estimación del $\mathrm{VO}_{2}$ máx (Kline, Porcari, Hintermeister, Freedson, Ward, McCarron, et al., 1987):

$\mathrm{VO}_{2}$ máx $=132.853-(0.0769 \times$ peso corporal en libras) $-0.3877 \times$ edad en años $+(6.315 \times$ puntaje de sexo $)$ - (3.2649 × tiempo en minutos para caminar 1 milla) - $(0,1565 \times$ frecuencia cardíaca en el final de la caminata). En cuanto al sexo, el valor 1 corresponde a hombres y el valor 0 es para mujeres.

\section{El índice de Calidad de Sueño de} Pittsburgh (ICSP): es un cuestionario que mide la calidad de sueño y sus alteraciones en el último mes (Buysse, Reynolds, Monk, Berman \& Kupfer, 1989). Se utilizó la versión del ICSP en castellano, utilizado en los estudios de países latinoamericanos (EscobarCórdoba \& Eslava-Schmalbach, 2005; Luna-Solis, Robles-Arana \& AgueroPalacios, 2015). El ICSP consta de 19 preguntas de autoevaluación que proporciona una puntuación global de la Calidad de Sueño y puntuaciones parciales de siete dimensiones del sueño (calidad subjetiva del sueño, latencia de sueño, duración del sueño, eficiencia habitual del sueño, perturbaciones extrínsecas del sueño, uso de medicación hipnótica y disfunción diurna). La puntuación de cada uno de los siete componentes oscila entre $O$ (no existe dificultad) y 3 (grave dificultad), tiene una medida de consistencia interna-a de Cronbach: $r=0,83$, demostrando ser una herramienta válida para evaluar trastornos de sueño y capaz de discriminar pacientes con diferente gravedad de los síntomas (Royuela \& Macías, 1997).

\section{Síntomas de Síndrome de Intestino Irritable relacionados con la calidad} de vida: para determinar posibles síntomas del SIl se aplicó el cuestionario "Best Score de 4 preguntas" (Spiegel, Naliboff, Mayer, Bolus \& Chang, 2006). Las respuestas a estas preguntas se miden en una escala en base a por- 
centaje: O (el mejor estado de salud) a 100 (el peor estado de salud). La calificación final asignada se puede usar como criterio de inclusión de pre-diagnóstico antes de entrar a una consulta de SII, es un instrumento corto, diseñado a partir de la medición de la calidad de vida relacionada con el SII (IBS-QQL score), encontrando un elevado grado de concordancia entre ambos instrumentos, sobre todo está recomendado para evaluar la gravedad ( $\mathrm{p}=0,007)$ y la frecuencia de deposiciones ( $p=0,05)$ por lo que puede ser una herramienta en la toma de decisiones en el escenario de la práctica clínica habitual (busy clinics) (Spiegel, et al., 2006; Spiegel, et al., 2010)

\section{Procedimiento}

Inicialmente, se contactó con los profesores de la clase de Educación Física para informarles del protocolo de participación en el estudio de sus alumnos; primero se midieron las variables antropométricas en el laboratorio de fisiología del ejercicio de la CURSPS-UPNFM, después se procedió al Ilenado de los cuestionarios "BEST-Score 4 points" y Cuestionario ICSP; se dispuso de 15 minutos para su contestación. Al siguiente día se realizó la prueba de caminar una milla en el polideportivo del departamento de Educación Física. Todas las pruebas fueron realizadas en el horario de clases en presencia de los maestros y fueron medidas por parte de los estudiantes de Educación Física, con una guía de los estándares necesarios, asimismo, el análisis de los datos fue realizado por un experto en estadística, para evitar el sesgo en los resultados del estudio.

\section{Análisis de los datos}

El análisis de los datos se realizó en función de la calificación del IMC y nivel del $\mathrm{VO}_{2}$ máx divididos por las tablas de interpretación de acuerdo al sexo y edad agrupados por terciles $<25 \%$, al 50\% y >75\%, indicando los niveles del $\mathrm{VO}_{2}$ máx de la siguiente manera: "nivel 1 = muy bajo", "nivel 2 = bajo" y "Nivel 3 = aceptable/bueno".

\section{Análisis estadístico}

Se realizó un análisis descriptivo representado con la media \pm desviación estándar. Se utilizó la prueba de Kolmogorov-Smirnov ( $n>50)$ para verificar que los datos presentaron distribución normal. Cuando se cumplió la normalidad se realizó la prueba de análisis factorial Anova para observar diferencias entre grupos con post hoc de Bonferroni; también se utilizó la correlación de Pearson para el estudio de relación de variables. Los análisis se llevaron a cabo utilizando SPSS para Windows (SPSS v.22.0, Chicago, IL, EE.UU.). El nivel de significación estadística se estableció en $\mathrm{p}<0,05$ con intervalos de confianza (95\%). 


\section{Resultado}

Tabla 1

Descripción del estado físico, $\mathrm{VO}_{2}$ máx, calidad del sueño y síntomas del (SII) en estudiantes universitarios de la UPNFM.

\begin{tabular}{lccc}
\hline Variable & Media/SD & Mínimo & Máximo \\
\hline Edad (años) & $25 \pm 9$ & 18 & 48 \\
\hline Talla (M) & $1,63 \pm 0,08$ & 1,46 & 1,79 \\
\hline Peso (Kg) & $68,6 \pm 18,5$ & 44,5 & 128 \\
\hline IMC (Kg/h 2) & $25,6 \pm 5,7$ & 17 & 40,9 \\
\hline Cintura (cm) & $82,6 \pm 15,7$ & 66 & 131 \\
\hline Cadera (cm) & $99,2 \pm 15,0$ & 76 & 126 \\
\hline ICC (cintura/cadera) & $0,83 \pm 0,08$ & 0,70 & 1,03 \\
\hline \% de masa grasa & $25,7 \pm 9,9$ & 6 & 40,1 \\
\hline Índice de Adiposidad Corporal (\%) & $30,3 \pm 6,0$ & 18,8 & 41,4 \\
\hline VO ${ }_{2}$ máx (ml/kg/min) & $34,5 \pm 9,8$ & 22 & 60 \\
\hline BEST-Score & $0,70 \pm 0,72$ & 0 & 2,7 \\
\hline Síntomas de SII (\%) & $17,6 \pm 18,0$ & 0 & 68,7 \\
\hline Índice de calidad del sueño & $0,68 \pm 0,64$ & 0 & 2 \\
\hline
\end{tabular}

La Tabla 1 presenta características del estado físico, $\mathrm{VO}_{2}$ máx, calidad del sueño y los síntomas del SII mediante la media \pm desviación estándar. Se observó un IMC que representa el sobrepeso según la calificación de la Organización Mundial de la Salud.
También, un $\mathrm{VO}_{2}$ máx con un nivel bajo en estudiantes universitarios que asisten a clase de Educación física; se muestra que no hay mucha dificultad para dormir $(0,68 /$ porcentaje = 22,6\%) y síntomas del SII muy baja $(0,70 /$ porcentaje $=17 \%)$. 
Vasquez, A. (2019). Asociación del sobrepeso y obesidad, consumo de oxígeno y calidad del sueño con los síntomas del intestino irritable en estudiantes universitarios de la UPNFM. Revista Ciencias de la Actividad Física UCM, N²0(2), julio-diciembre, 1-19. DOI: http://doi.org/10.29035/rcaf.20.2.6

Tabla 2

Comparación del Vo $\mathrm{O}_{2}$ máx, calidad del sueño y síntomas del (SII) en función del IMC en estudiantes universitarios de la UPNFM.

\begin{tabular}{lcccc}
\hline Grupos & Normopeso & Sobrepeso & Obesidad & \\
\cline { 1 - 4 } Variables & Media/DS & Media/DS & Media/DS & P valor \\
\hline Edad (años) & $22 \pm 7$ & $27 \pm 9$ & $26 \pm 10 \zeta$ & 0,192 \\
\hline IMC (Kg/h^2) & $20,6 \pm 1,5$ & $\mathbf{2 7 , 5} \pm 1,5^{*}$ & $32,0 \pm 5,51 \zeta$ & 0,001 \\
\hline Cintura (cm) & $73,8 \pm 9,6$ & $86,1 \pm 6,5$ & $93,6 \pm 21,91$ & 0,000 \\
\hline Cadera (cm) & $91,0 \pm 12,0$ & $101,6 \pm 5,1^{*}$ & $110,4 \pm 18,71 \zeta$ & 0,000 \\
\hline ICC (cintura/cadera) & $0,81 \pm 0,07$ & $0,85 \pm 0,06$ & $0,85 \pm 0,11$ & 0,316 \\
\hline \% de masa grasa & $19,7 \pm 7,4$ & $\mathbf{2 7 , 0} \pm 11,2 *$ & $34,6 \pm 3,71 \zeta$ & 0,000 \\
\hline Índice de Adiposidad Corporal (\%) & $26,2 \pm 4,2$ & $\mathbf{3 1 , 5} \pm \mathbf{4 , 0 *}$ & $35,8 \pm 5,61 \zeta$ & 0,000 \\
\hline VO, máx (ml/kg/min) & $36,1 \pm 11,0$ & $36,4 \pm 10,4$ & $29,9 \pm 4,7$ & 0,103 \\
\hline BEST-Score & $0,4 \pm 0,4$ & $0,5 \pm 0,5$ & $1,3 \pm 0,91 \zeta$ & 0,000 \\
\hline Síntomas de SII (\%) & $11,5 \pm 10,7$ & $12,5 \pm 12,9$ & $33,3 \pm 23,11 \zeta$ & 0,000 \\
\hline Índice de calidad del sueño & $0,5 \pm 0,5$ & $0,8 \pm 0,7$ & $0,7 \pm 0,2$ & 0,291 \\
\hline N = Sujetos & $\mathrm{N}=25$ & $\mathrm{~N}=19$ & $\mathrm{~N}=18$ & \\
\hline
\end{tabular}

* Diferencias significativas entre grupos normopeso y sobrepeso, 1 diferencias entre grupos normopeso y obesidad y $\zeta$ diferencias entre grupos de sobrepeso y obesidad.

La Tabla 2 muestra diferencias por grupos en función del IMC representado con la media \pm desviación estándar. Se observa que el VO $\mathrm{O}_{2}$ máx no presenta diferencias entre los grupos, asimismo, no existió diferencia en la calidad del sueño, mostrando que no hay dificultad para dormir entre los estudiantes. En los síntomas del SII (\%), se muestran diferencias entre sujetos (normopeso/sobrepeso) vs obesidad, observándose diferencias de $21,8 \%$ y $22,8 \%$, respectivamente, siendo mayores los síntomas del SII en sujetos que presentan obesidad. 
Tabla 3

Comparación del estado físico, calidad del sueño y síntomas del (SII) en función del consumo máximo de oxígeno en estudiantes universitarios de la UPNFM.

\begin{tabular}{lcccc}
\hline Grupos & Nivel 1 & Nivel 2 & Nivel 3 & \\
\cline { 1 - 4 } Variables & Media/DS & Media/DS & Media/DS & P valor \\
\hline Edad (años) & $26 \pm 9$ & $26 \pm 10$ & $23 \pm 8$ & 0,543 \\
\hline IMC (Kg/h^2) & $28,0 \pm 6,4$ & $24,7 \pm 5,8$ & $24,2 \pm 4$ & 0,072 \\
\hline Cintura (cm) & $91,6 \pm 18,3$ & $78,1 \pm 7,0 *$ & $77,5 \pm 12,91$ & 0,005 \\
\hline Cadera (cm) & $106,1 \pm 13,4$ & $98,3 \pm 7,2$ & $94,3 \pm 16,71$ & 0,028 \\
\hline ICC (cintura/cadera) & $0,86 \pm 0,10$ & $0,80 \pm 0,07$ & $0,83 \pm 0,0$ & 0,109 \\
\hline \% de masa grasa & $30,3 \pm 7,1$ & $29,3 \pm 7,1$ & $20,9 \pm 10,91$ & 0,002 \\
\hline Índice de Adiposidad Corporal (\%) & $33,3 \pm 4,8$ & $29,8 \pm 5,1$ & $28,4 \pm 6,61$ & 0,036 \\
\hline VO máx (ml/kg/min) & $26,5 \pm 3,5$ & $31,6 \pm 3,5$ & $41,8 \pm 9,3$ & 0,000 \\
\hline BEST-Score & $0,8 \pm 0,4$ & $1,0 \pm 0,9$ & $0,4 \pm 0,71$ & 0,029 \\
\hline Síntomas de SII (\%) & $21,9 \pm 10,4$ & $26,3 \pm 24,8$ & $11,1 \pm 17,91$ & 0,029 \\
\hline Índice de calidad del sueño & $0,8 \pm 0,6$ & $0,9 \pm 0,5$ & $0,4 \pm 0,61$ & 0,043 \\
\hline N = Sujetos & 22 & 13 & 27 & \\
\hline
\end{tabular}

* Diferencias entre grupos del Nivel 1 y Nivel 2, 1 diferencias entre grupos del Nivel 1 y Nivel 3 y $\zeta$ diferencias entre grupos del Nivel 2 y Nivel 3.

La Tabla 3 muestra diferencias significativas en función del $\mathrm{VO}_{2}$ máx representado con la media \pm desviación estándar. Se observan diferencias en el estado físico entre grupos del $\mathrm{Ni}$ vel 1 vs Nivel 2, siendo mayor el perímetro de cintura en el grupo Nivel 1. Un mayor número de diferencias se observan en comparación del grupo Nivel 1 vs Nivel 3 en las variables: Cintura, Cadera, \% de Masa Grasa y IAC.
También se muestran diferencias en IPCS del 13\%, siendo mejor la calidad del sueño por parte del grupo Nivel 3 , pero los dos grupos se encuentran en niveles estándares para conciliar el sueño. Se pueden observar niveles más alto de síntomas del SII en sujetos del grupo Nivel 1 vs grupo Nivel 3 con diferencias del 10,8\%, siendo mayor los síntomas del SII en los sujetos que tienen $\mathrm{VO}_{2}$ máx más bajo. 
Vasquez, A. (2019). Asociación del sobrepeso y obesidad, consumo de oxígeno y calidad del sueño con los síntomas del intestino irritable en estudiantes universitarios de la UPNFM. Revista Ciencias de la Actividad Física UCM, N²0(2), julio-diciembre, 1-19. DOI: http://doi.org/10.29035/rcaf.20.2.6

Tabla 4

Correlaciones entre obesidad, $\mathrm{VO}_{2}$ máx, calidad del sueño y síntomas del (SII) en estudiantes universitarios de la UPNFM.

\begin{tabular}{lcccccccc}
\hline Variables & Edad & IMC & ICC & \% de Grasa & IAC & VO $_{2}$ máx & Síntomas SII & IPSC \\
\hline Edad & 1 & $0,29^{*}$ & $0,35^{* *}$ & $0,36^{* *}$ & $0,27^{*}$ & $-0,37^{* *}$ & $-0,23$ & 0,19 \\
\hline IMC & - & 1 & $0,26^{*}$ & $0,63^{* *}$ & $0,68^{* *}$ & $-0,29^{*}$ & $0,57^{* *}$ & $0,30^{*}$ \\
\hline ICC & - & - & 1 & $-0,03$ & $-0,17$ & 0,02 & $-0,28^{*}$ & $-0,13$ \\
\hline \% de Grasa & - & - & - & 1 & $0,64^{* *}$ & $-0,76^{* *}$ & $0,60^{* *}$ & $0,56^{* *}$ \\
\hline IAC & - & - & - & - & 1 & $-0,55^{* *}$ & $0,49^{* *}$ & $0,43^{* *}$ \\
\hline VO ${ }_{2}$ máx & - & - & - & - & - & 1 & $-0,38^{* *}$ & $-0,52^{* *}$ \\
\hline Síntomas SII & - & - & - & - & - & - & 1 & $0,47^{* *}$ \\
\hline IPSC & - & - & - & - & - & - & - & 1 \\
\hline
\end{tabular}

*p: valor $<0,05 y^{* *}$ p: valor $<0,01$ estadísticamente significativo.

La Tabla 4 muestra las correlaciones entre las diferentes variables del estudio representadas con el valor rPearson. Se observa que estudiantes que puntúan mayor en el SII, son los que poseen un IMC ( $r=0,51 ; p=0,00)$, Masa Grasa \% ( $r=0,60 ; p=0,00)$ y IAC $(r=0,49 ; p=0,00)$ mayor. Además, se muestra que los que tienen mayor Masa Grasa \% ( $r=0,56 ; p=0,00), I A C$ $(r=0,43 ; p=0,00)$, síntomas del SII ( $r$ $=0,47 ; p=0,00)$ y VO 2 máx $(r=-0,52$; $\mathrm{p}=0,00)$, obtuvieron peor calidad del sueño. Todos los resultados tienen correlación moderada, excepto el $\mathrm{VO}_{2}$ máx, con el \% de masa grasa ( $r=-0,76 ; p=0,00)$, lo cual parece ser la variable del sobrepeso y obesidad que más afecta al SII, IPCS y VO $\mathrm{O}_{2}$ máx.

\section{Discusión}

El principal hallazgo del estudio fue la propuesta de asociación que tiene el $\mathrm{VO}_{2}$ máx con el padecimiento de síntomas de SII y la calidad del sueño en estudiantes universitarios. La prevalencia en este estudio de síntomas evaluados con BEST-Score fue bajo (17,6 \pm 18,0 \%); estos resultados son apoyados por el estudio de Vargas-Matos, Ng-Sueng, Flores-Arriaga, Beltran-Flores, Lema-Correa, Piscoya, et al., 2015, donde se muestra una prevalencia baja en estudiantes universitarios (12,4\%). Contrario al estudio de Ibrahim (2016), donde observó una prevalencia de síntomas relativamente alta de SII por el estrés que generan los estudiantes; no obstante, en nuestro estudio, algunos estudiantes presentaron síntomas mayores (68,7\%). 
También se muestra una calidad del sueño poco alterada con valores medios 22,6 \pm 21,3\%, lo que concuerda con el estudio de Sierra, Jiménez \& Domingo, 2002, donde la muestra estudiada alcanzó valores bajos de calidad del sueño (30\%), contrario al estudio de Monterrosa, Ulloque \& Carriazo (2014), donde la prevalencia de la muestra de la población tuviera un valor promedio (48,5\%), siendo este un valor más bajo comparado con el máximo valor en nuestro estudio, $66,6 \%$ en la puntuación global del índice de Pittsburgh, asimismo, estos autores encuentran que los estudiantes universitarios con insomnio y malos dormidores presentan una mala calidad de vida.

En cuanto al estado físico, solo existen diferencias entre sujetos normopeso-sobrepeso vs obesidad en los síntomas de SII (11,5 \pm 10,7 / 12,5 \pm $12,9)$ vs $(33,3 \pm 23,1)$. Estos datos pueden ser apoyados por el estudio de Aasbrenn, Lydersen \& Farup (2018) donde se encontró que los síntomas intestinales mejorados se asociaron inversamente con un IMC reducido. Aunque el IMC es contradictorio para algunos científicos pues, según Kubo, Fujiwara, Shiba, Kohata, Yamagami, Tanigawa, et al., 2011, el IMC puede ser menor en sujetos con SII y la prevalencia no suele ser alta en individuos obesos, sin embargo, Hingorjo, Zehra, Hasan \& Qureshi (2017) proponen la evaluación del estado físico como seguimiento en la prevención del desa- rrollo de enfermedades crónicas no transmisibles como el SII.

Respecto a las relaciones de SII con el $\mathrm{VO}_{2}$ máx, se justifican en estudios como el de Niemyjska, Ukleja \& Lawinski (2015) que en una muestra de estudiantes universitarios discriminó los trastornos del movimiento intestinal por niveles de actividad física, siendo más frecuentes en los que realizaban poca actividad física. El aumento de actividad física mejora el $\mathrm{VO}_{2}$ máx y los síntomas gastrointestinales del SII y deben utilizarse como una modalidad de tratamiento primario (Johannesson, Simrén, Strid, Bajor \& Sadik, 2011). La última revisión sobre buenas prácticas en el tratamiento de SII (Fukudo, Kaneko, Akiho, Inamori, Endo, Okumura, et al., 2014), pone de manifiesto la recomendación de la actividad física con un nivel de evidencia científica $B, y$ es que el aumento de actividad física en sujetos con síntomas de SII puede hacer que mantengan una absorción de oxígeno relativamente estable (Johannesson et al., 2015).

Uno de los mecanismos fisiológicos que explica el efecto positivo del aumento del $\mathrm{VO}_{2}$ máx en sujetos con síntomas de SII, son las interacciones cerebro-intestino en la disminución del estrés que puede influir favorablemente en la plasticidad cerebral (Dishman, Berthoud, Booth, Cotman, Edgerton, Fleshner, et al., 2006). El estrés induce la exageración de la respuesta neuroendocrina y las alteraciones vis- 
cerales perceptivas (Posserud, Agerforz, Ekman, Björnsson, Abrahamsson \& Simrén, 2004). Es por esta razón que el SII se encuentra asociado a la ansiedad, depresión y estrés (Cerecedo, 2011). El aumento de $\mathrm{VO}_{2}$ máx mediante la actividad física está relacionado de manera inversa sobre síntomas depresivos y, por lo tanto, puede proteger contra el deterioro de síntomas del SII (Johannesson, et al., 2011).

Otro estudio evaluó los cambios del estrés oxidativo y biomarcadores inflamatorios demostrando que la actividad física de intensidad baja a moderada atenúa favorablemente la inflamación de las citoquinas plasmáticas (IL-7 $\beta$, IL-6, IL-8, IL-10 y TNF- $\alpha$ ) adenosina desaminasa, estrés oxidativo (XO, MDA y NO) y antioxidantes potenciados (SOD, CAT y GSH-PX). Estas alteraciones se correlacionan con mejoras prometedoras en síntomas de SII (Hajizadeh-Maleki, Tartibian, Mooren, FitzGerald, Krüger, Chehrazi, et al., 2018); otro estudio también muestra cómo el aumento del $\mathrm{VO}_{2}$ máx está relacionado con el Nervio Vago (NV), importante en el equilibrio del sistema nervioso simpático/parasimpático responsable de la homeóstasis, siendo un componente clave de los ejes neuro-inmunes e intestino cerebral a través de una comunicación bidireccional entre el cerebro y el tracto gastrointestinal (GI) (Bonaz, Sinniger \& Pellissier, 2017). Se sabe que la acti- vidad física y el entrenamiento físico pueden ejercer un efecto estimulante sobre la actividad vagal y marcadores inflamatorios (Sloan, McCreath, Tracey, Sidney, Liu \& Seeman, 2007), sin embargo, la actividad física intensa puede causar un desequilibrio por altos niveles cardiorrespiratorios (Laukkanen, Lakka, Rauramaa, Kuhanen, Venäläinen, Salonen, et al., 2001). Por lo tanto, será interesante observar en futuros estudios si un aumento del $\mathrm{VO}_{2}$ máx debería ser enfocado por dosis-respuesta en sujetos con síntomas de SII (Johannesson, et al., 2015).

En cuanto a la calidad del sueño se asoció con la actividad física relacionada con el $\mathrm{VO}_{2}$ máx ( $r=-0,52 ; p=$ $0,00)$; estos datos son apoyados por el estudio de Mota \& Vale (2010) donde se encontró que los sujetos con un menor $\mathrm{VO}_{2}$ máx tienen peor calidad del sueño, y es que entre mayor sea el $\mathrm{VO}_{2}$ máx, mayores seran los efectos positivos sobre el sistema nervioso central y periférico (Blanco-Centurion \& Shiromani, 2006). Es importante considerar que la calidad del sueño y el SII están totalmente relacionados por múltiples vías, entre las que se destacan en este estudio: el equilibrio del sistema nervioso autónomo (SNA) y biomarcadores inflamatorios (Chennaoui, Arnal, Sauvet, \& Léger, 2015), y los efectos que puede tener la actividad física regular y aumento de la condición física relacionado con el $\mathrm{VO}_{2}$ máx. 


\section{Limitaciones}

Se necesitan llevar a cabo estudios metodológicos más rigurosos para comprender la relación e influencia del $\mathrm{VO}_{2}$ máx con la calidad del sueño y el SII, ya que, sin mediciones de la tasa de metabolismo basal, función cardíaca, control de la glucosa, estado de ánimo y función inmune, es realmente difícil discriminar los mecanismo directos e indirectos implicados en cambios inducidos por actividad física. En la última revisión de ejercicio y microbiota intestinal (Mailing, Allen, Buford, Fields \& Woods, 2019) se explica el papel que podría cumplir el ejercicio físico en relación con la enfermedad inflamatoria intestinal y el efecto de reducción de los síntomas que esto conlleva, además, se propone realizar más ensayos clínicos controlados para identificar el tipo de actividad, frecuencia e intensidad del ejercicio físico que mejoren y/o ayuden al control de la enfermedad. Además, otra limitación es la no estimación directa del $\mathrm{VO}_{2}$ máx con análisis de gases; sin embargo, el protocolo de Rockport se acepta como una aproximación confiable para medir el $\mathrm{VO}_{2}$ máx (Weiglein, Herrick, Kirk \& Kirk, 2011) en estudiantes universitarios.

\section{Recomendaciones}

Realizar estudios con más cuestionarios que evalúen la calidad de vida relacionada con los síntomas del SII como las (FBDSI, IBS-QOL, EQ5D11, CDC-4), de igual manera, como se ex- plicó en los mecanismos fisiológicos del $\mathrm{VO}_{2}$ máx con la actividad vagal, se propone medir el equilibrio simpático/parasimpático a través de Variabilidad de la Frecuencia Cardiaca durante las intervenciones con actividad física, además de controlar el tipo de alimentación de los sujetos, ya que puede condicionar la prevalencia de sufrir la enfermedad. También se propone realizar un estudio experimental de control randomizado, para observar como la actividad física puede provocar cambios en síntomas del SII, calidad del sueño y $\mathrm{VO}_{2}$ máx, aquí es donde se destaca los cambios inducidos por las clases de Educación Física a nivel universitario en la UPNFM.

Finalmente, es necesario prestar mayor atención a esta patología (SII). Así, se pueden plantear estrategias en las universidades que permitan prevenir o reducir la presencia de factores de riesgo. Si bien esta patología no tiene una cura específica, es posible mejorar la calidad de vida, evitando que se vean afectados el entorno personal y profesional en el futuro de los estudiantes universitarios (Foster, et al., 2014).

\section{Conclusión}

Los resultados indicaron que los estudiantes universitarios de la UPNFM que tienen un mayor consumo de oxígeno máximo podrían conciliar mejor el sueño y mostrar valores más bajos en la puntuación de síntomas de síndrome de intestino irritable re- 
lacionada con la calidad de vida. Con este estudio se propone realizar estudios de ensayos clínicos enfocados en la asociación del $\mathrm{VO}_{2}$ máx con el síndrome del colon irritable y la calidad del sueño en diferentes poblaciones.

\section{REFERENCIAS BIBLIOGRÁFICAS}

Aasbrenn, M., Lydersen, S., \& Farup, P. G. (2018). A Conservative Weight Loss Intervention Relieves Bowel Symptoms in Morbidly Obese Subjects with Irritable Bowel Syndrome: A Prospective Cohort Study. Journal of Obesity, 2018, Article ID 3732753, 1-9. Recuperado de https://doi. org/10.1155/2018/3732753

Alaqeel, M. K., Alowaimer, N. A., Alonezan, A. F., Almegbel, N. Y., \& Alaujan, F. Y. (2017). Prevalence of irritable bowel syndrome and its association with anxiety among medical students at King Saud bin Abdulaziz University for health sciences in Riyadh. Pakistan Journal of Medical Sciences, 33(7), 3336. Recuperado de https://doi. org/10.12669/pjms.331.12572

Alvero-Cruz, J., Correas Gómez, L., Ronconi, M., Fernández Vázquez, R., \& Porta i Manzañido, J. (2011). La bioimpedancia eléctrica como método de estimación de la composición corporal: normas prácticas de utilización. Revista Andaluza de Medicina del Deporte, 4(4), 167-174. Recuperado de https://doi.org/10.1016/S18887546(14)70058-9

Bergman, R. N., Stefanovski, D., Buchanan, T. A., Sumner, A. E., Reynolds, J. C., Sebring, N. G., ... Watanabe, R. M. (2011). A better index of body adiposity. Obesity, 19(5), 1083-1089. Recuperado de https://doi.org/10.1038/ oby.2011.38

Blanco-Centurion, C. A. \& Shiromani, P. J. (2006). Beneficial effects of regular exercise on sleep in old F344 rats. Neurobiology of Aging, 27(12), 1859-1869. Recuperado de https://doi.org/10.1016/j. neurobiolaging.2005.10.009

Bonaz, B., Sinniger, V. \& Pellissier, S. (2017). The vagus nerve in the neuro-immune axis: Implications in the pathology of the gastrointestinal tract. Frontiers in Immunology 8, 1-74. Recuperado de https://doi.org/10.3389/ fimmu.2017.01452 
Browning, L. M., Hsieh, S. D., \& Ashwell, M. (2010). A systematic review of waist-to-height ratio as a screening tool for the prediction of cardiovascular disease and diabetes: 05 could be a suitable global boundary value. Nutrition Research Reviews, 23(2), 247269. Recuperado de https://doi. org/10.1017/s0954422410000144

Buysse, D. J., Reynolds, C. F., Monk, T. H., Berman, S. R. \& Kupfer, D. J. (1989). The Pittsburgh Sleep Quality Index: a new instrument for psychiatric practice and research. Psychiatry research, 28(2), 193-213. Recuperado de https://doi.org/10.1016/01651781(89)90047-4

Cañón, M., Ruiz, A. J., Rondón, M., \& Alvarado, J. (2017). Prevalence of irritable bowel syndrome and health-related quality of life in adults aged 18 to 30 years in a Colombian university: An electronic survey. Annals of Gastroenterology, 30(1), 6775. Recuperado de https://doi. org/10.20524/aog.2016.0093

Cerecedo, R. B. (2011). Síndrome de intestino irritable en estudiantes de medicina. Revista de la Facultad de Medicina de la UNAM., 54(3), 4-12. Recuperado de http://www.scielo.org.mx/scielo. php?script $=$ sci_arttext\&pid $=$ s0026-17422011000300002\&lng $=$ es\&tlng $=$ es .
Chennaoui, M., Arnal, P. J., Sauvet, F., \& Léger, D. (2015). Sleep and exercise: A reciprocal issue? Sleep Medicine Reviews 20, 5972. Recuperado de https://doi. org/10.1016/j.smrv.2014.06.008

Cortés, X., Roset, M., Barrull, C., Sais, C., \& Raya, A. (2016). Coste del síndrome del intestino irritable con predominio de estreñimiento en ESP. Pharmacoeconomics Spanish Research Articles, 13(1), 1-8. Recuperado de https://doi. org/10.1007/s40277-015-0048-0

De Schryver, A. M., Keulemans, Y. C., Peters, H. P., Akkermans, L. M., Smout, A. J., De Vries, W. R., \& Van Berge-Henegouwen, G. P. (2005). Effects of regular physical activity on defecation pattern in middle-aged patients complaining of chronic constipation. Scandinavian Journal of Gastroenterology, 40(4), 422429. Recuperado de https://doi. org/10.1080/00365520510011641

Dishman, R. K., Berthoud, H. R., Booth, F. W., Cotman, C. W., Edgerton, V. R., Fleshner, M. R., ... Zigmond, M. J. (2006). Neurobiology of exercise. Obesity, 74(3), 345356. Recuperado de https://doi. org/10.1038/oby.2006.46

Drossman, D. A. (2006). The Functional Gastrointestinal Disorders and the Rome III Process. Gastroenterology 130(5), 1377-1390. Recuperado de https://doi. org/10.1053/j.gastro.2006.03.008 
Escobar-Córdoba, F., \& Eslava-Schmalbach, J. (2005). Validación colombiana del índice de calidad de sueño de Pittsburgh. Revista de Neurología, 40(3), 150-155. Recuperado de https:// doi.org/rn2004320 [pii]

Foster, C., Hillsdon, M., Thorogood, M., \& Kaur, A. (2014). Interventions for promoting physical activity. Cochrane Database Syst Rev, 9(1), 1-90. Recuperado de https://doi.org/10.1002/14651858. CD003180.pub2

Fukudo, S., Kaneko, H., Akiho, H., Inamori, M., Endo, Y., Okumura, T., ... Shimosegawa, T. (2014). Evidence-based clinical practice guidelines for irritable bowel syndrome. Journal of Gastroenterology, 50(1), 11-30. Recuperado de https://doi.org/10.1007/ s00535-014-1017-0

Hajizadeh Maleki, B., Tartibian, B., Mooren, F. C., FitzGerald, L. Z., Krüger, K., Chehrazi, M., \& Malandish, A. (2018). Low-tomoderate intensity aerobic exercise training modulates irritable bowel syndrome through antioxidative and inflammatory mechanisms in women: Results of a randomized controlled trial. Cytokine, 102, 18-25. Recuperado de https://doi.org/10.1016/j. cyto.2017.12.016
Hingorjo, M. R., Zehra, S., Hasan, Z., \& Qureshi, M. A. (2017). Cardiorespiratory fitness and its association with adiposity indices in young adults. Pakistan Journal of Medical Sciences, 33(3), 659664. Recuperado de https://doi. org/10.12669/pjms.333.12294

Ibrahim, N. K. (2016). A systematic review of the prevalence and risk factors of irritable bowel syndrome among medical students. The Turkish Journal of Gastroenterology, 27(1), 1016. Recuperado de https://doi. org/10.5152/tjg.2015.150333

Johannesson, E., Ringstrom, G., Abrahamsson, H., \& Sadik, R. (2015). Intervention to increase physical activity in irritable bowel syndrome shows long-term positive effects. World journal of gastroenterology, 27(2), 600608. Recuperado de https://doi. org/10.3748/wjg.v21.i2.600

Johannesson, E., Simrén, M., Strid, H., Bajor, A., \& Sadik, R. (2011). Physical Activity Improves Symptoms in Irritable Bowel Syndrome: A Randomized Controlled Trial. American Journal of Gastroenterology, 706(5), 915-922. Recuperado de https://doi. org/10.1038/ajg.2010.480 
Kline, G. M., Porcari, J. P., Hintermeister, R., Freedson, P. S., Ward, a, McCarron, R. F., ... Rippe, J. M. (1987). Estimation of $\mathrm{VO}_{2} \mathrm{max}$ from a one-mile track walk, gender, age, and body weight. Medicine and science in sports and exercise 19(3),253-259. Recuperado de https://www.ncbi. nlm.nih.gov/pubmed/3600239

Kubo, M., Fujiwara, Y., Shiba, M., Kohata, Y., Yamagami, H., Tanigawa, T., ... Arakawa, T. (2011). Differences between risk factors among irritable bowel syndrome subtypes in Japanese adults. Neurogastroenterology and motility: the official journal of the European Gastrointestinal Motility Society, 23(3), 249-254. Recuperado de https://doi.org/10.1111/ j.1365-2982.2010.01640.x

Laukkanen, J. a, Lakka, T. a, Rauramaa, R., Kuhanen, R., Venäläinen, J. M., Salonen, R., \& Salonen, J. T. (2001). Cardiovascular fitness as a predictor of mortality in men. Archives of internal medicine, 767(6), 825-831. Recuperado de https://doi.org/10.1001/archinte.161.6.825

Ledo-Varela, M. T., de Luis Román, D. A., González-Sagrado, M., IzaolaJauregui, O., Conde-Vicente, R., \& Aller de la Fuente, R. (2011). Características nutricionales y estilo de vida en universitarios. Nutricion Hospitalaria, 26(4), 814-818. Recuperado de https:// doi.org/10.3305/nh.2011.26.4.5156
Luna-Solis, Y., Robles-Arana, Y., \& Aguero-Palacios, Y. (2015). Validación del índice de calidad de sueño de Pittsburgh en una muestra peruana. Anales de Salud Mental, 37(2), 23-30. Recuperado de http://www.insm. gob.pe/ojsinsm/index.php/Revistal/article/view/15/14

Mailing, L. J., Allen, J. M., Buford, T. W., Fields, C. J., \& Woods, J. A. (2019). Exercise and the Gut Microbiome: A Review of the Evidence, Potential Mechanisms, and Implications for Human Health. Exercise and Sport Sciences Reviews, 47(2), 75-85. Recuperado de https://doi.org/10.1249/ JES.0000000000000183

Monterrosa Castro, A., Ulloque Caamaño, L., \& Carriazo Julio, S. (2014). Calidad del dormir, insomnio y rendimiento académico en estudiantes de medicina. Duazary, 77(2), 85 - 97. Recuperado de https://doi. org/10.21676/2389783X.814

Mota, J., \& Vale, S. (2010). Associations between sleep quality with cardiorespiratory fitness and BMI among adolescent girls. American Journal of Human Biology, 22(4), 473-475. Recuperado de https://doi.org/10.1002/ ajhb.21019 
Niemyjska, S., Ukleja, A., \& Lawinski, M. (2015). Evaluation Of Irritable Bowel Syndrome Symptoms Amongst Warsaw University Students. Polski przeglad chirurgiczny, 87(5), 252-259. Recuperado de https://doi.org/10.1515/ pjs-2015-0050

Posserud, I., Agerforz, P., Ekman, R., Björnsson, E. S., Abrahamsson, H., \& Simrén, M. (2004). Altered visceral perceptual and neuroendocrine response in patients with irritable bowel syndrome during mental stress. Gut, 53(8), 1102-1108. Recuperado de https://doi.org/10.1136/ gut.2003.017962

Quigley, E. M. M., Abdel-Hamid, H., Barbara, G., Bhatia, S. J., Boeckxstaens, G., De Giorgio, R., ... Tzeuton, C. (2012). A global perspective on irritable bowel syndrome: A consensus statement of the world gastroenterology organisation summit task force on irritable bowel syndrome. Journal of Clinical Gastroenterology, 46(5), 356-366. Recuperado de https://doi.org/10.1097/ MCG.Ob013e318247157c

Royuela Rico, A., \& Macías Fernández, J. A. (1997). Propiedades clinimétricas de la versión castellana del Cuestionario de Pittsburgh. Vigilia-Sueño, 9(2), 81-94.
Sierra, J., Jiménez, C., \& Domingo, J. (2002). Calidad del sueño en estudiantes universitarios: Importancia de la higiene del sueño. Salud Mental, 25(6), 35-43. Recuperado de https://www.medigraphic.com/cgi-bin/new/resumen.cgi? IDARTICULO = 17415

Sloan, R. P., McCreath, H., Tracey, K. J., Sidney, S., Liu, K., \& Seeman, T. (2007). RR interval variability is inversely related to inflammatory markers: the CARDIA study. Molecular medicine (Cambridge, Mass.), 13(3-4), 178184. Recuperado de https://doi. org/10.2119/2006-00112.Sloan

Song, B. K., Cho, K. O., Jo, Y., Oh, J. W., \& Kim, Y. S. (2012). Colon transit time according to physical activity level in adults. Journal of Neurogastroenterology and Motility, 18(1), 64-69. Recuperado de https://doi.org/10.5056/ jnm.2012.18.1.64

Spiegel, B. M. R., Bolus, R., Harris, L. A., Lucak, S., Chey, W. D., Sayuk, G., ... Chang, L. (2010). Characterizing abdominal pain in IBS: Guidance for study inclusion criteria, outcome measurement and clinical practice. Alimentary Pharmacology and Therapeutics, 32(9), 1192-1202. Recuperado de https://doi.org/10.1171/j.13652036.2010.04443.x 
Spiegel, B. M. R., Naliboff, B., Mayer, E., Bolus, R., \& Chang, L. (2006). Development and initial validation of a concise point-of-care severity index in IBS: The «B.E.S.T.» questionnaire. Gastroenterology 730, s1040.

Talley, N. J., Gabriel, S. E., Harmsen, W. S., Zinsmeister, A. R., \& Evans, R. W. (1995). Medical costs in community subjects with irritable bowel syndrome. Gastroenterology, 109(6), 1736-1741. Recuperado de https://doi.org/10.1016/00165085(95)90738-6

Vargas-Matos, I., Ng-Sueng, L., Flores-Arriaga, J., Beltrán-Flores, S., Lema-Correa, M., Piscoya, A., \& Mayta-Tristán, P. (2017). Superposición del síndrome de intestino irritable y dispepsia funcional basados en criterios ROMA III en estudiantes de medicina de una universidad privada de Lima, Perú. Revista de Gastroenterología del Perú, 35(3), 219-25. Recuperado de http://www.revistagastroperu.com/index.php/rgp/article/ view/107/104
Weiglein, L., Herrick, J., Kirk, S., \& Kirk, E. P. (2011). The 1-mile walk test is a valid predictor of $\mathrm{VO}_{2}$ max and is a reliable alternative fitness test to the 1.5-mile run in U.S. Air Force males. Military medicine, 776(6), 669-673. Recuperado de https://doi.org/10.7205/ MILMED-D-10-00444

\section{Dirección para correspondencia}

Aldo Alfonso Vasquez Bonilla Máster en Promoción de la Salud Mediante Actividad Física.

Departamento de Educación Física, Universidad Pedagógica Nacional Francisco Morazán.

San Pedro Sula, Honduras.

Número de registro en ORCID:

0000-0002-5319-086X

Contacto:

aldovasquez1994@hotmail.com

Recibido: 09-06-2019

Aceptado: 06-11-2019 